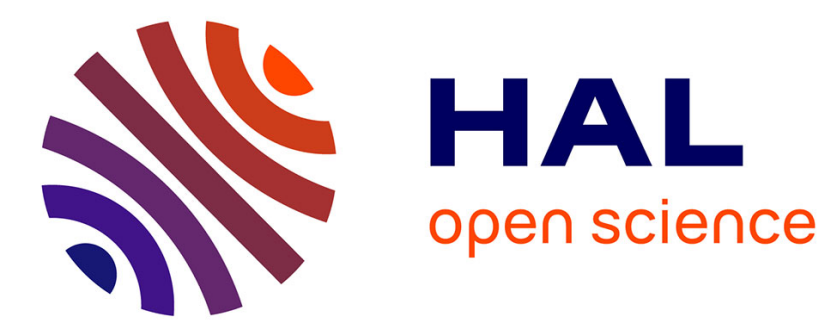

\title{
Discriminateur de maxima à circuit simulant la fonction diode idéale
}

\author{
A. Chehikian
}

\section{To cite this version:}

A. Chehikian. Discriminateur de maxima à circuit simulant la fonction diode idéale. Revue de Physique Appliquée, 1967, 2 (2), pp.101-108. 10.1051/rphysap:0196700202010100 . jpa-00242768

\section{HAL Id: jpa-00242768 https://hal.science/jpa-00242768}

Submitted on 1 Jan 1967

HAL is a multi-disciplinary open access archive for the deposit and dissemination of scientific research documents, whether they are published or not. The documents may come from teaching and research institutions in France or abroad, or from public or private research centers.
L'archive ouverte pluridisciplinaire HAL, est destinée au dépôt et à la diffusion de documents scientifiques de niveau recherche, publiés ou non, émanant des établissements d'enseignement et de recherche français ou étrangers, des laboratoires publics ou privés. 


\title{
DISGRIMINATEUR DE MAXIMA \\ A GIRGUIT SIMULANT LA FONGTION DIODE IDÉALE
}

\author{
Par A. CHEHIKIAN, \\ École Nationale Supérieure d'Électronique de Grenoble.
}

\begin{abstract}
Résumé. - On décrit un appareillage " traduisant " les maxima d'un signal électrique par des impulsions dont l'amplitude et la position dans le temps correspondent à celles des maxima.

Le principe de cette discrimination de maxima repose sur les propriétés des circuits à diodes et capacités. L'emploi des circuits à diodes réelles manquant de précision pour cette fonction, nous proposons un montage simulant les propriétés d'une diode idéale dont les qualités peuvent être ajustées selon la précision et la réponse désirées.
\end{abstract}

On expose enfin les résultats obtenus.

\begin{abstract}
We describe a device which translates the maxima of an electrical signal into pulses, the amplitude and position of which are identical with those of the maxima.

The principle of this discrimination of maxima is based on the properties of diode and capacitor circuits. Because of the use of real diodes for this function induces inaccuracy, we propose a circuit which simulates ideal diode properties, the quality of which can be adjusted to obtain the wanted precision and response.

We finally describe the results we obtained.
\end{abstract}

\section{INTRODUCTION}

Le dispositif que nous nous proposons de décrire fait partie d'un ensemble destiné à discriminer des signaux se propageant sur un faisceau de voies de transmission (fibres nerveuses par exemple). Chaque voie étant dotée d'une vitesse de propagation caractéristique, nous utiliserons comme critère de discrimination cette vitesse de propagation.

Le problème se ramène donc à la mesure du temps de parcours des signaux entre deux points de prélèvement. Les données de ce problème sont les suivantes :

- Les signaux électriques transportés par chacune des voies présentent une réponse temporelle de même type que celui représenté ci-dessous :

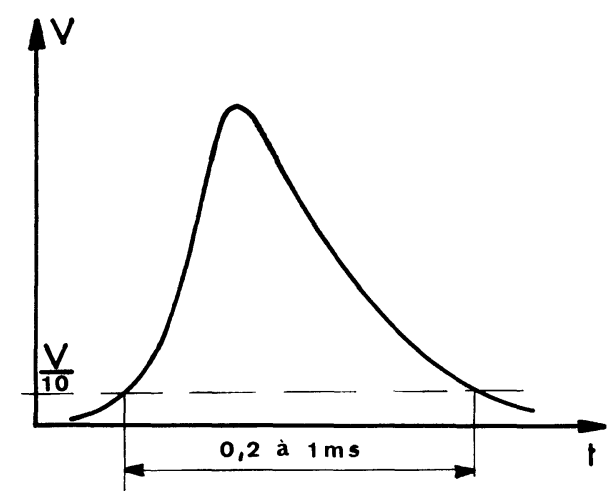

FIG. 1. - Forme type des signaux à traiter.
- Les temps de parcours à mesurer s'étagent entre 0,1 et 1 milliseconde. Ils sont donc d'un ordre de grandeur comparable à celui de la largeur des signaux.

- La nature des voies de transmission est telle qu'il est difficile d'effectuer des prélèvements identiques d'un point à l'autre d'une voie. Par ailleurs, les signaux sont d'amplitude différente selon la voie qui les transporte. La mesure du temps de parcours devra donc être indépendante de l'amplitude des signaux.

- Cependant, l'amplitude des signaux pouvant aussi être caractéristique d'une voie de transmission, il est souhaitable, pour un complément d'analyse ultérieur en amplitude, de conserver, dans le signal convenablement mis en forme, la donnée de cette amplitude.

Toutes ces considérations nous ont donc amené à réaliser la transformation suivante : faire correspondre au signal à traiter une impulsion de largeur très faible par rapport à celle du signal primitif, dont l'amplitude est la même (à un facteur près) que celle de ce signal et dont la position dans le temps est celle du maximum de ce signal. Il nous faut donc réaliser la détection précise de ce maximum.

Dans la suite de cet article, nous utiliserons les symboles suivants :

$V_{\mathrm{D}} \quad:$ tension de coude de diode (c'est-à-dire tension au-delà de laquelle la résistance de la diode peut être considérée comme très faible, sinon nulle). 
$I_{\mathrm{c}}, I_{\mathrm{e}}, I_{\mathrm{b}}$ : représentent respectivement le courant de collecteur, d'émetteur et de base du transistor.

$I_{\mathrm{e}_{0}}, I_{\mathrm{e}_{0}}$ : dans le transistor, sont respectivement le courant inverse de saturation de la jonction collecteur-base et émetteur-base.

$\alpha_{N} \quad$ : représente la fraction des porteurs injectés par l'émetteur dans la base et qui atteignent la région de transition de la jonction collecteur-base.

$\alpha_{\mathrm{I}} \quad:$ représente la fraction des porteurs injectés par le collecteur dans la base et qui atteignent la région de transition de la jonction émetteur-base.

Dans le fonctionnement normal du transistor (c'est-à-dire jonction émetteur-base polarisée en direct et jonction collecteurbase en inverse), le gain en courant global $I_{\mathrm{c}} / I_{\mathrm{e}}=\alpha$ est alors égal à $\alpha_{\mathrm{N}}$.

$\beta \quad$ : rapport du courant de collecteur au courant d'émetteur :

$$
\beta=I_{\mathrm{c}} / I_{\mathrm{b}}=\alpha / 1-\alpha .
$$

$\lambda \quad$ : représente la quantité $q / \eta K T$ :

$q$ : charge de l'électron,

$K$ : constante de Boltzmann,

$T$ : température absolue,

$\eta$ : constante dont la valeur est comprise entre 1 et 2 pour le silicium et qui tend vers 1 pour le germanium. A la température de $3000^{\circ} \mathrm{K}$ et pour $\eta=1$ : $1 / \lambda=26$ millivolts.

\section{DETECTION D'UN MAXIMUM}

2.1. Principe de la détection. Circuit de base. Une solution simple pour résoudre le problème de la détection du passage et un signal par un maximum consiste à détecter le passage par zéro du signal « dérivé ». Nous avons écarté cette solution car elle conduit à une imprécision qui est fonction de l'amplitude et de la forme du signal.

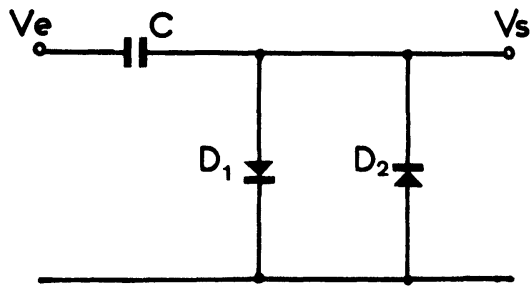

FIG. 2. - Circuit de base.

Nous avons préféré adopter une méthode qui consiste à aligner le maximum sur un niveau de tension fixe et détecter ensuite le passage du signal par un seuil situé au-dessous de ce niveau. La première partie de cette opération est réalisée par le circuit de base décrit à la figure 2 et dérivé du très classique circuit de " clamping ».
La figure 4 détaille la forme du signal de sortie en supposant que les diodes $\mathrm{D}_{1}$ et $\mathrm{D}_{2}$ ont la caractéristique idéalisée représentée à la figure 3 .

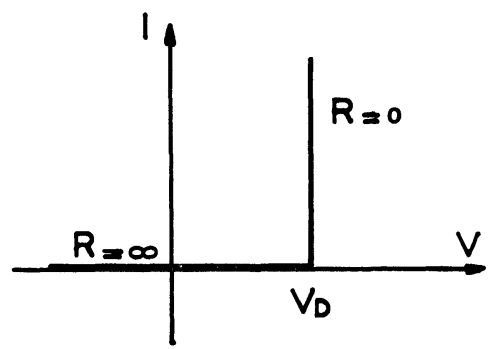

FIG. 3. - Caractéristique de la diode idéale.

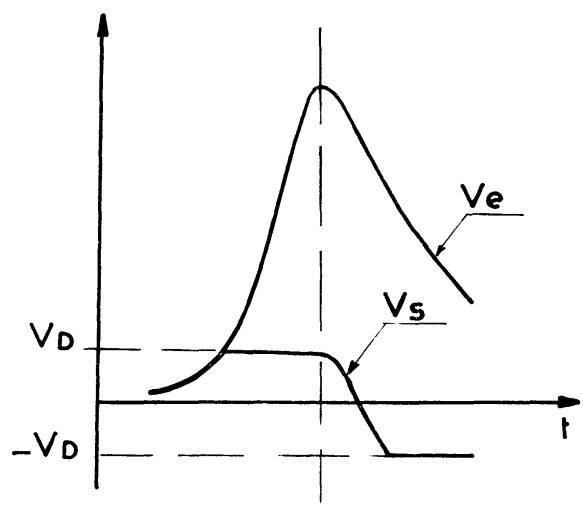

FIG. 4. - Réponse du circuit de base.

Remarquons que, dans ce circuit, $\mathrm{D}_{1}$ a un rôle primordial, $\mathrm{D}_{2}$ ne servant qu'à décharger le condensateur après la détection du maximum. Par ailleurs, l'intérêt de ce montage est que le fonctionnement est indépendant de l'amplitude du signal pourvu que celle-ci soit supérieure à la tension de coude de la diode $\mathrm{D}_{1}$; de même, sa largeur n'intervient pas dans le fonctionnement du circuit. Cependant, ces proprié-

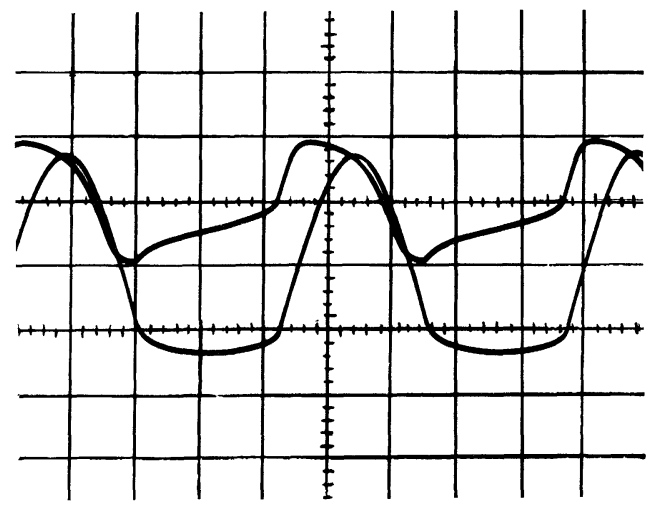

FIG. 5. - Réponse du circuit de base réalisé à l'aide de la diode réelle. Traces supérieure et inférieure $0,2 \mathrm{~V} / \mathrm{div}$; balayage $0,2 \mathrm{~ms} /$ div. La photographie met en évidence le mauvais fonctionnement dû à la caractéristique courbe de la diode. 
tés ne se conservent pas lorsqu'on utilise des diodes réelles comme le montre la photographie de la figure 5. Nous nous sommes donc attaché à réaliser un dispositif ayant la caractéristique $I(V)$ idéale représentée à la figure 3.

2.2. Diode à caractéristique idéale. - 2.2.1. PRINGIPE. - Une diode « idéale » pourrait se caractériser comme un élément dont la résistance est nulle ou très faible lorsqu'on applique à ses bornes une tension supérieure à la tension de coude $V_{\mathrm{D}}$ et devient infinie dans le cas inverse.

Un tel élément peut, en particulier, être réalisé par un transistor $\mathrm{T}$ dont on commandera convenablement la conduction entre collecteur et émetteur, grâce à l'intervention d'un amplificateur de gain A. Le schéma de cette réalisation est représenté à la figure 6 .

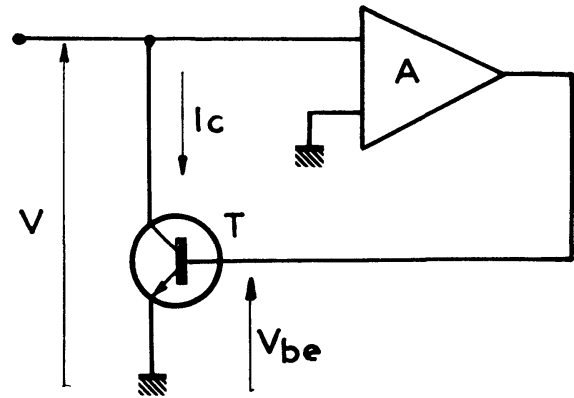

FIG. 6. - Schéma de principe du circuit réalisant la fonction " diode idéale ".

Dans notre étude théorique, nous supposerons que l'impédance d'entrée de l'amplificateur A est infinie et son impédance de sortie nulle.

Un calcul simple permet de prévoir la relation $I_{\mathrm{c}}(V)$ $\mathrm{du}$ transistor $\mathrm{T}$ dont on rappelle les équations qui en définissent le fonctionnement en courant continu :

$I_{\mathrm{e}}=\frac{-I_{\mathrm{e}_{0}}}{1-\alpha_{\mathrm{N}} \alpha_{\mathrm{I}}}\left(\mathrm{e}^{\lambda V_{\mathrm{be}}}-1\right)+\frac{\alpha_{\mathrm{I}} I_{\mathrm{c}_{0}}}{1-\alpha_{\mathrm{N}} \alpha_{\mathrm{I}}}\left(\mathrm{e}^{-\lambda V_{\mathrm{cb}}}-1\right)$

$$
\begin{gathered}
I_{\mathrm{c}}=\frac{\alpha_{\mathrm{N}} I_{\mathrm{e}_{\mathrm{o}}}}{1-\alpha_{\mathrm{N}} \alpha_{\mathrm{I}}}\left(\mathrm{e}^{\lambda V_{\mathrm{be}}}-1\right)-\frac{I_{\mathrm{c}_{\mathrm{o}}}}{1-\alpha_{\mathrm{N}} \alpha_{\mathrm{I}}}\left(\mathrm{e}^{-\lambda V_{\mathrm{cb}}}-1\right) \\
I_{\mathrm{c}}+I_{\mathrm{b}}+I_{\mathrm{e}}=0 \\
V_{\mathrm{be}}+V_{\mathrm{ec}}+V_{\mathrm{cb}}=0
\end{gathered}
$$

En tenant compte du schéma de la figure 6 , nous pouvons écrire :

$$
V_{\mathrm{be}}=A V+V_{0}
$$

De ces relations, nous tirons, en supposant que $A \gg 1$ :

$$
I_{\mathrm{c}}=\frac{\alpha_{\mathrm{N}} I_{\mathrm{e}_{\mathrm{o}}}-I_{\mathrm{c}_{0}}}{1-\alpha_{\mathrm{N}} \alpha_{\mathrm{I}}}\left(\mathrm{e}^{\lambda A\left(V+\frac{V_{0}}{A}\right)}-1\right) .
$$

Comparons cette relation à celle d'une diode classique qui est :

$$
I=I_{\mathrm{s}}\left(\mathrm{e}^{\lambda V}-1\right)
$$

Nous voyons que ces deux relations sont du même type ; cependant, deux termes les différencient :

- d'une part, l'existence d'une tension $-V_{0} / A$ en dessous de laquelle la « diode » est effectivement bloquée (seul subsiste un courant de fuite);

- d'autre part, le coefficient de $\lambda$. Or, on sait que la région de transition entre l'état bloqué et l'état conducteur sera d'autant plus faible que ce coefficient est grand.

Par le choix de $V_{0}$ et $A$, il nous est donc possible d'obtenir une caractéristique $I(V)$ se rapprochant aussi près que l'on veut de la caractéristique d'une diode idéale, dont on maîtrise en plus la valeur de la tension de coude.

2.2.2. RÉalisation PRATiQue DU Dispositif DE « DIODE A GARAGTÉRISTIQUE IDÉALE ». - Le schéma du dispositif est donné ci-dessous ( $f g .7$ ).

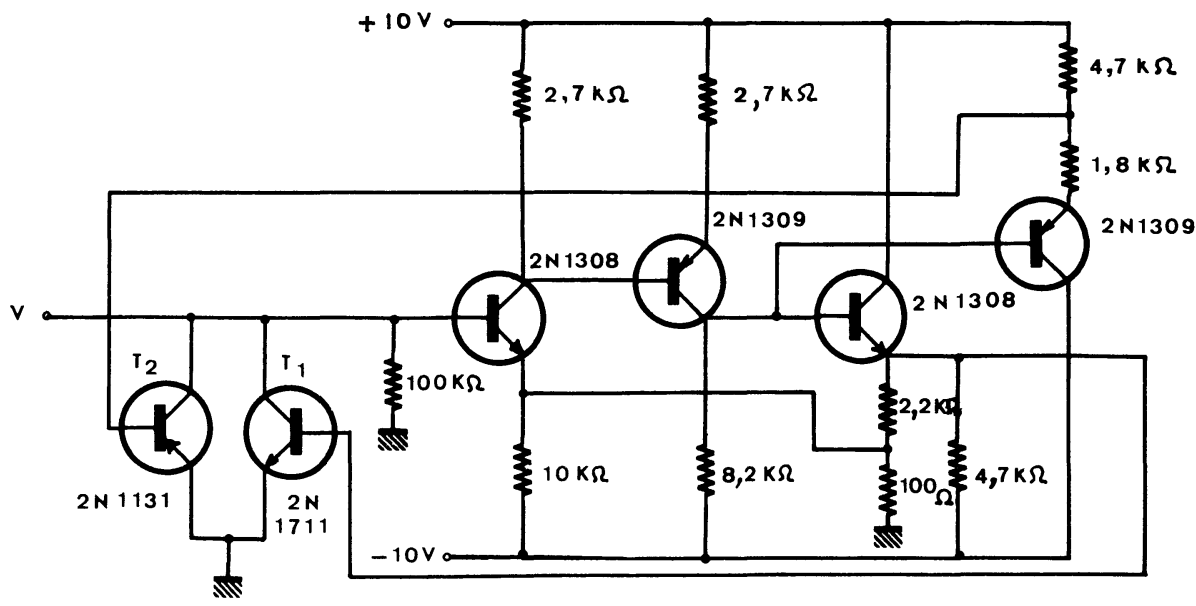

FIG. 7. - Schéma du circuit réalisant la double fonction " diode idéale ". 
Les deux diodes du circuit de base de la figure 2 sont réalisées à partir de deux transistors connectés selon le schéma de principe précédemment décrit. La fonction « amplificateur A » est assurée par un amplificateur à courant continu doté d'un circuit de contre-réaction.

Nous repérerons tout ce qui a trait au circuit de commande du transistor $T_{1}$ par l'indice 1 et ce qui concerne le circuit de commande de $\mathrm{T}_{2}$ par l'indice 2 . Selon les définitions précédentes des symboles, on aurait dans ce montage :

$$
\begin{array}{ll}
A_{1}=20 & A_{2}=15 \\
V_{01}=-2,6 \mathrm{~V} & V_{02}=+0,6 \mathrm{~V}
\end{array}
$$

$V_{01}$ et $A_{1}$ ont été choisis de façon à obtenir le meilleur effet « diode » possible pour les tensions $V_{\mathrm{e}}$ positives ;

$V_{02} \quad$ a été déterminé pour assurer la plus petite tension de coude de l'effet " diode » pour les $V_{\mathrm{e}}$ négatifs possible afin de permettre une décharge correcte du condensateur.

Nous avons testé ce dispositif par un relevé de la caractéristique d'entrée tracée à l'oscilloscope dont la photographie de la figure 8 montre l'image.

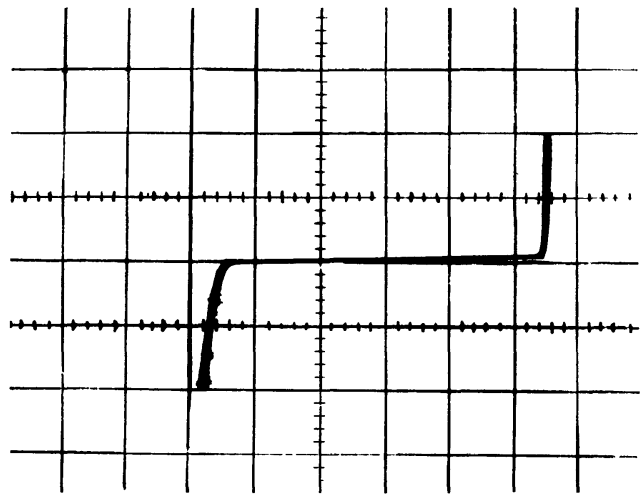

FIG. 8. - Caractéristique d'entrée $I(V)$ du circuit réalisant la double fonction " diode idéale ", relevée à l'oscilloscope.

Déviation verticale $1 \mathrm{~mA} / \mathrm{div}$; déviation horizontale $50 \mathrm{mV} /$ div.

Afin de comparer les qualités du dispositif et celles d'un montage équivalent réalisé à l'aide de diodes du commerce, nous avons superposé les caractéristiques des deux montages sur la photographie de la figure 9 .

Enfin, nous avons testé la réponse du circuit de base de la figure 2 , réalisé à l'aide de ce dispositif. Cette réponse est représentée ci-après ( $f g .10$ ).

On peut comparer cette photographie à celle de la

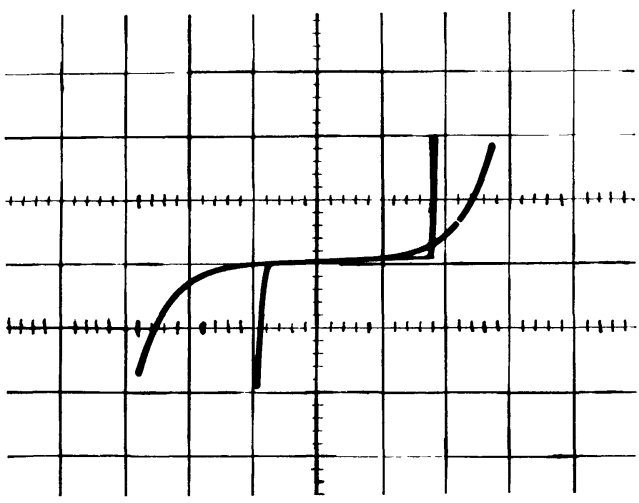

FIG. 9. - Caractéristiques comparées du circuit de la figure 7 et d'un montage à diodes classiques.

Déviation verticale $1 \mathrm{~mA} / \mathrm{div}$; déviation horizontale $100 \mathrm{mV} /$ div.

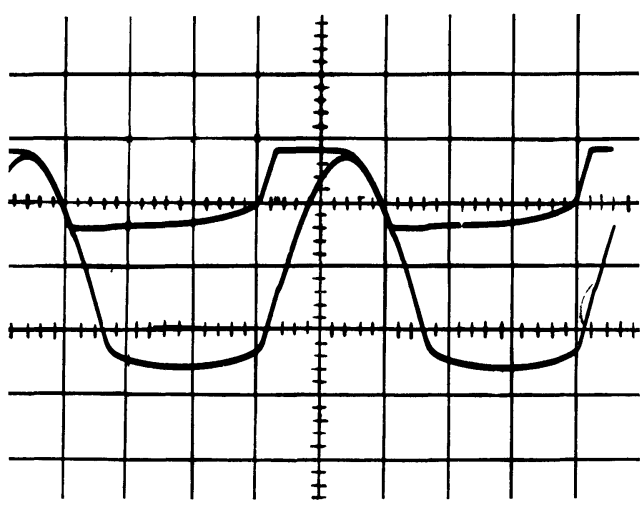

FIG. 10. - Réponse du circuit réalisé à l'aide du montage de la figure 7. Comparée à la photograghie de la figure 4 , celle-ci fait ressortir l'amélioration apportée au fonctionnement du circuit de base.

figure 5. Elle montre que l'information sur la position du maximum est conservée et se traduit effectivement par une rupture de pente du signal de sortie au passage de $V_{\text {e }}$ par un maximum.

2.3. Détecteur de maximum. - 2.3.1. RÉALISATION PRATIQUE. - Le schéma du détecteur de maximum est donné par la figure 11.

On reconnaît en section I le circuit de base réalisé à l'aide du dispositif " diode idéale » précédemment décrit. Il est suivi en sections II et III d'un ensemble d'amplificateurs à seuil dont la fonction est de détecter le passage du signal par un niveau situé en dessous de la tension de coude de la « diode " $\mathrm{D}_{\mathbf{1}}$.

Les oscillogrammes de la figure 12 illustrent les formes des tensions en divers points du montage pour un signal d'entrée identique à celui représenté sur la photographie de la figure 10. 


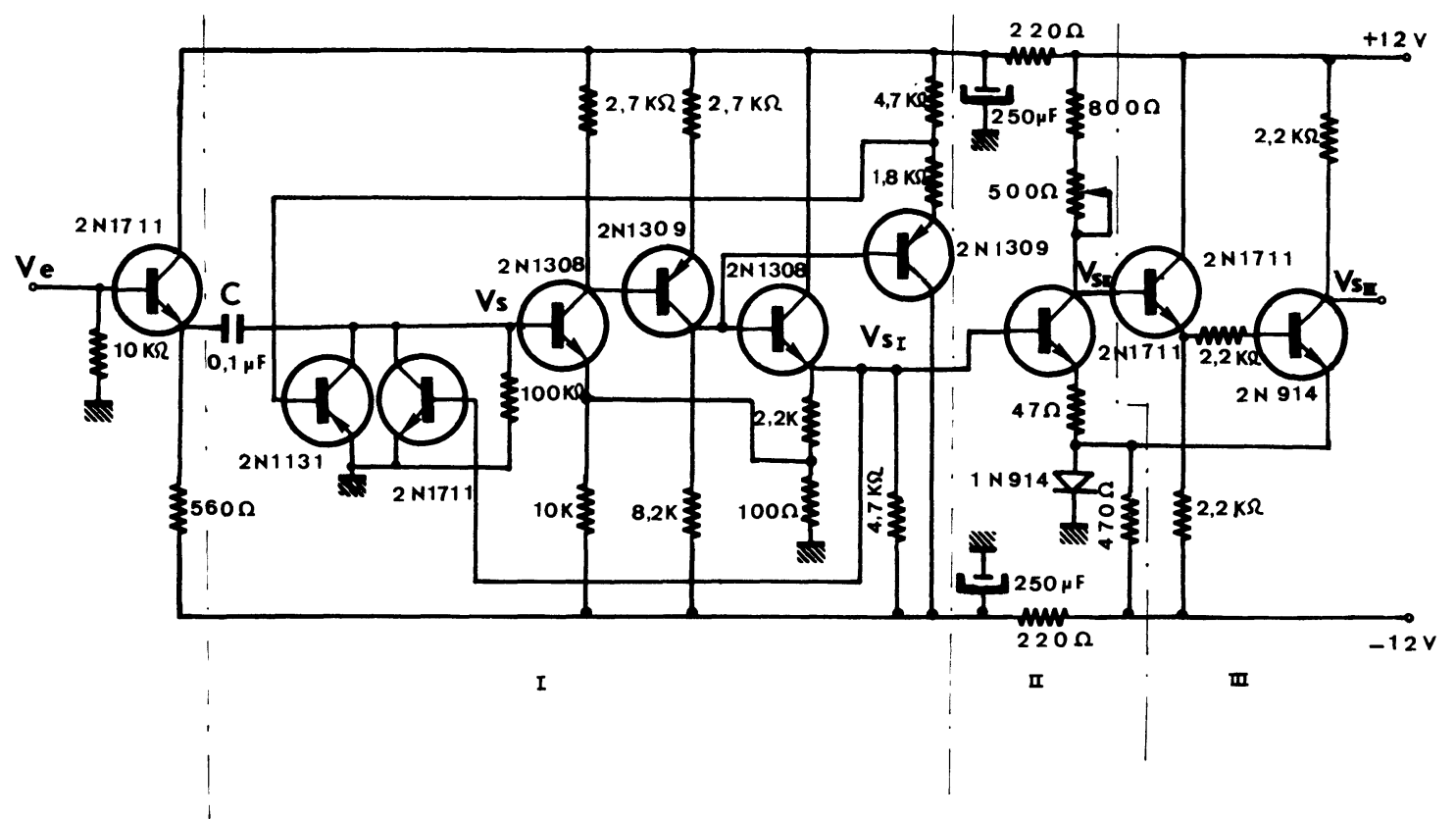

FIG. 11. - Détecteur de maxima ; réalisation pratique.

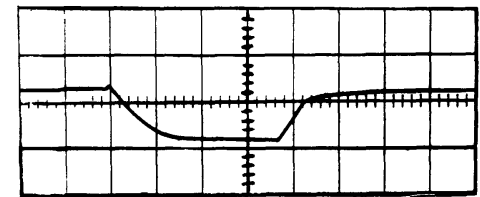

FIG. 12 a. - Oscillogramme relevé sur les "diodes idéales " Sensibilité $0,2 \mathrm{~V} /$ div.

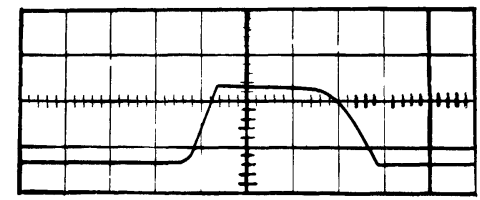

Fig. $12 b$. - Oscillogramme relevé à la sortie de 1'amplificateur de la fonction " diode idéale ». La forme du signal est identique à la précédente mais à niveau plus élevé et aligné sur le niveau $+0,6 \mathrm{~V}$ - sensibilité $2 \mathrm{~V} / \mathrm{div}$.

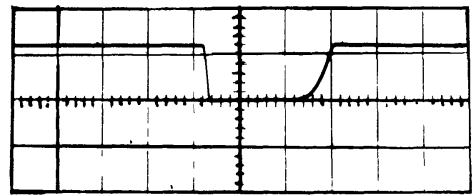

FIG. 12 c. - Oscillogramme relevé à la sortie du premier amplificateur à seuil. Seule la partie positive du signal de la figure $12 b$ a été amplifiée - sensibilité $10 \mathrm{~V} /$ div.

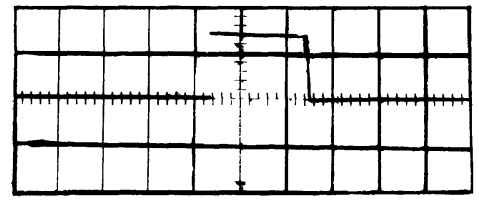

Fig. $12 d$. - Oscillogramme relevé à la sortie du détecteur. Le front de descente du signal traduit le passage du signal d'entrée par un maximum - sensibilité $10 \mathrm{~V} /$ div, Balayage $0,1 \mathrm{~ms} / \mathrm{div}$.

FIG. 12. - Oscillogrammes relevés en divers points du schéma de la figure 11. Le signal d'entrée est identique à celui de la figure 10.

2.3.2. RÉsultats DE MESURE. - a) Mesure sur un signal unique. Précision. - Afin de déterminer la précision de l'ensemble, nous avons effectué des mesures en utilisant, comme signal d'entrée, un signal sinusoïdal redressé de largeur et d'amplitude variables.

Le dispositif étant destiné à traiter des signaux de largeur comprise entre 0,1 et $1 \mathrm{~ms}$ et d'amplitude variable dans un rapport de 10 , nous avons limité le domaine de mesure à ces valeurs.
Nous caractérisons la précision par le rapport $\eta=t_{0} / T$ défini sur la figure 13 .

Le résultat de ces mesures est consigné dans le graphique de la figure 14 . Nous pouvons remarquer sur ce graphique qu'au-delà d'une certaine amplitude, pour une largeur donnée, la précision est indépendante de l'amplitude. Le front de descente du détecteur se trouve donc retardé d'une valeur constante $t_{0}$ par rapport à la position du maximum vrai. Nous voyons 


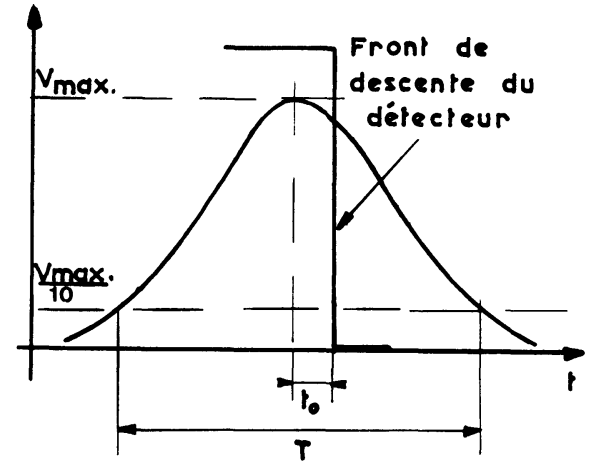

FIG. 13. - L'écart entre la position réelle du maximum et la position du front de descente définit la précision du détecteur.

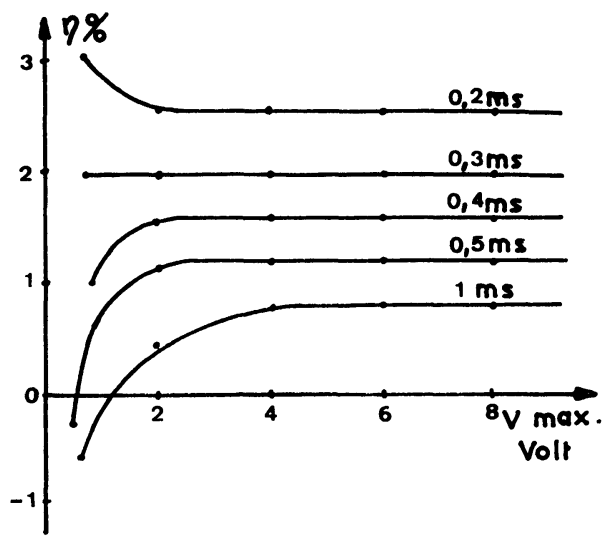

Fig. 14. - Précision du détecteur en fonction de l'amplitude et de la largeur du signal à traiter. Le graphique montre qu'au-delà d'une certaine amplitude et pour une largeur donnée l'imprécision du détecteur se traduit par un retard constant.

là une propriété fort intéressante lorsqu'il s'agit de faire, comme nous nous le proposons, des mesures précises de temps de parcours.

Remarque. - Aux faibles amplitudes, on note des anomalies dues, pour les signaux larges, à une décharge non négligeable du condensateur dans la résistance d'entrée de l'amplificateur et pour les signaux de faible largeur à un déphasage de l'ensemble amplificateur d'attaque + condensateur $\mathrm{C}$.

b) Détection de maxima successifs. - Nous avons vu au paragraphe 2.3.1 que le détecteur de maximum est constitué :

- par un dispositif qui ramène le maximum à un niveau de tension limite $V_{\mathrm{D} 1}$,

- par un dispositif dont le seuil $S$ est inférieur à $V_{\mathrm{D} 1}$ d'une valeur $\varepsilon$.

L'atteinte de ce seuil dans le sens croissant réalise l' « armement » du détecteur ; le passage ultérieur dans le sens décroissant « déclenche » le front de descente du détecteur. Ceci montre qu'il n'est pas nécessaire, après passage par un maximum, que le signal d'entrée s'annule pour qu'on puisse détecter le maximum suivant. Il suffit pour cela que l'opération d' « armement » ait eu lieu.

La figure 15 explicite :

- une forme de signal à traiter comportant plusieurs maxima ;

- le signal obtenu aux bornes des « diodes »;

- la réponse du détecteur.

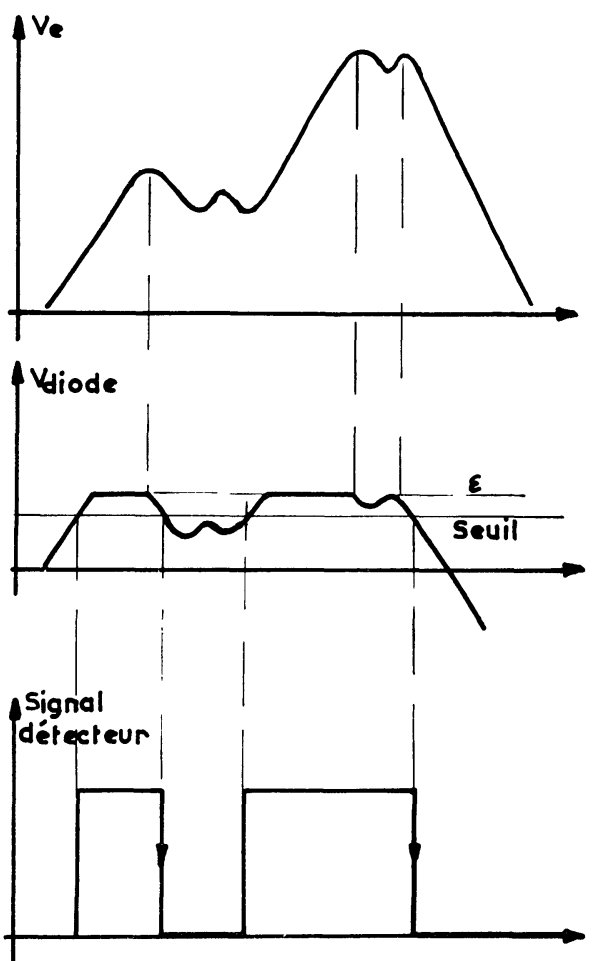

Fig. 15. - Comportement du détecteur devant un signal comportant plusieurs maxima.

Nous pouvons constater que deux maxima seulement ont été détectés (fronts de descente fléchés). En effet :

- le premier maximum a été détecté normalement ainsi que le quatrième ;

- le second ne l'a pas été car le détecteur n'a pas été « armé » lors de la recroissance du signal;

- le troisième non plus car le détecteur, bien qu' «armé », n’a pas été déclenché.

Cette propriété pourra être mise à profit pour détecter plusieurs signaux superposés, pourvu que la superposition donne lieu à des maxima tels que l'écart d'amplitude entre un maximum et le minimum suivant soit supérieur à la valeur $\left(V_{\mathrm{D}_{\mathbf{1}}}-S\right)$. De la même façon, le bruit superposé au signal donne lieu à des maxima qui peuvent être détectés intempestivement. Il est donc utile d'étudier l'immunité au bruit du détecteur. 
2.3.3. Immunité AU BRUIT. - L'aptitude du bruit à créer des maxima non désirables et détectables sur le signal tient essentiellement aux pentes relatives du signal et du bruit.

Nous avons donc superposé au signal utile un signal de bruit de forme quasi triangulaire (dont on peut facilement lier la largeur $\theta$ à la base et le spectre de fréquence) et nous avons mesuré le rapport signal/bruit minimal pour un fonctionnement non perturbé du détecteur (un seul maximum détecté).

Le résultat de ces mesures est consigné dans les graphiques de la figure 16. Il ressort de ces mesures que :

- pour un rapport $T / \theta$ donné, le rapport signal/bruit est indépendant de l'amplitude et de $T$;

- le rapport signal/bruit minimal croît rapidement avec $T / \theta$.

Il est donc impératif de limiter la bande passante des amplificateurs à la valeur suffisante pour transmettre correctement le signal à traiter.
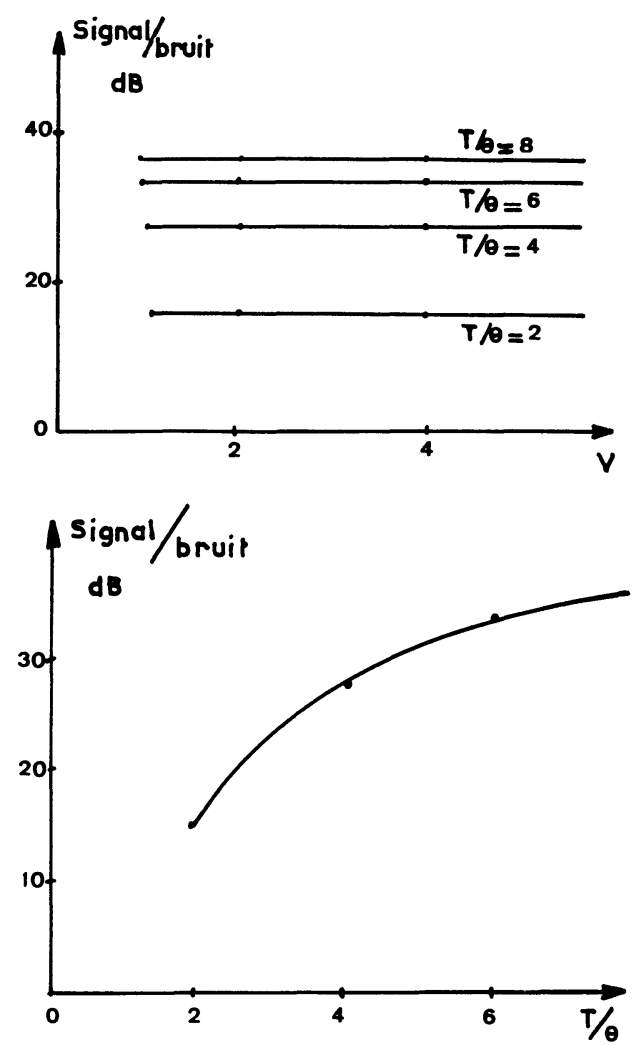

FIG. 16. - Immunité au bruit du détecteur : $T$ représente la largeur du signal ; $\theta$ représente la largeur du signal parasite.

\section{ENSEMBLE DE MISE EN FORME}

Le détecteur de maximum que nous venons de décrire n'est qu'un élément de l'ensemble de mise en forme dont le but est d'associer au signal d'entrée une impulsion calibrée en largeur. La position dans le temps de cette impulsion est celle du maximum du signal d'entrée et son amplitude est l'amplitude maximale de ce signal. Le schéma fonctionnel de l'ensemble de mise en forme est donné à la figure 17.

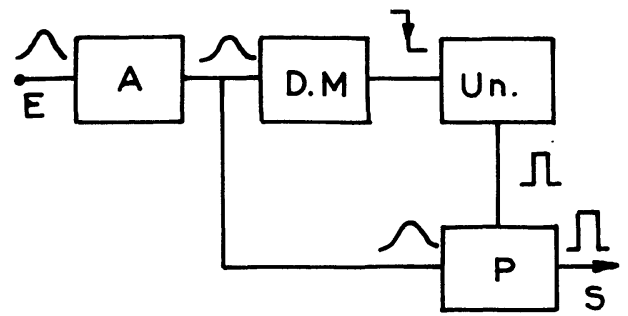

Fig. 17. - Schéma de principe de l'ensemble

$\mathrm{E}$ entrée.

A amplificateur d'entrée.

D.M détecteur de maximum.

Un univibrateur.

$\mathrm{P}$ porte.

$\mathrm{S} \quad$ sortie mise en forme.

L'ensemble de mise en forme n'est constitué que de fonctions classiques sans particularité aucune. Le détecteur de maximum déclenche un univibrateur qui commande l'ouverture de la porte sur laquelle on applique le signal d'entrée. A la sortie de la porte, on obtient ainsi une impulsion dont la largeur est celle de l'impulsion de l'univibrateur et dont l'amplitude est celle du signal. Les photographies de la figure 18 montrent des exemples de signaux soumis au traitement ainsi que les signaux résultants.
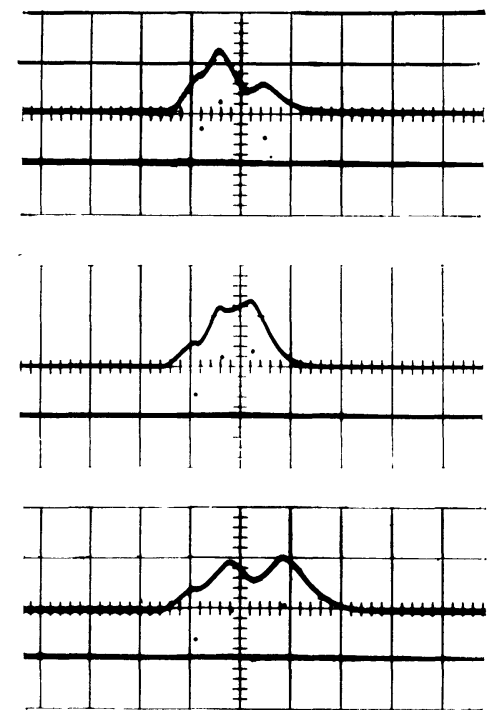

Fig. 18. - Exemples de signaux soumis au traitement.

Trace supérieure signal d'entrée $0,1 \mathrm{~V} /$ div.

Trace inférieure signal résultant $1 \mathrm{~V} / \mathrm{div}$.

Balayage $0,2 \mathrm{~ms} /$ div.

Seuls les sommets des impulsions sont visibles et se traduisent sur la photographie par des points. 
Performances. - Nous résumons ci-après les performances de cet ensemble de mise en forme que nous avons particulièrement étudiées :

— Signal d'entrée : largeur 0,1 à $1 \mathrm{~ms}$, amplitude $50 \mathrm{mV}$ à $1 \mathrm{~V}$.

- Impédance d'entrée : $10 \mathrm{k} \Omega$.

- Signal de sortie : largeur réglable de $10 \mu \mathrm{s}$ à $100 \mu \mathrm{s}$,

- Impédance de sortie : $50 \Omega$. amplitude $10 . V_{\mathrm{e}}$.

Il est cependant possible d'étendre le domaine de largeur ainsi que la dynamique d'amplitude des signaux à traiter, et ce par des modifications aisées. Elles ont trait essentiellement au circuit détecteur de maximum.

En ce qui concerne la largeur des signaux d'entrée, on pourra, en adaptant la capacité du condensateur de détection, déplacer le domaine de fonctionnement aussi bien vers les fréquences basses qu'élevées. On remarque toutefois que, vers les fréquences élevées, avec les circuits décrits, on sera limité par la bande passante des amplificateurs et, en particulier, l'amplificateur de l' « effet diode » $(50 \mathrm{kHz})$.

En ce qui concerne la dynamique d'amplitude, deux méthodes sont utilisables. On pensera d'abord à augmenter le gain des amplificateurs d'entrée. Toutefois, cela nécessiterait l'utilisation d'amplificateurs pouvant délivrer des signaux de très grande amplitude. On sera amené en conséquence à modifier les caractéristiques des « diodes » et ce, en augmentant le gain de l'amplificateur de l' « effet diode ».

Bien que nous ne l'ayons pas particulièrement étudiée, nous avons eu recours à cette dernière méthode qui nous a donné toute satisfaction.

Manuscrit reçu le 22 décembre 1966.

\section{BIBLIOGRAPHIE}

EBERS (J. J.), Large signal behavior of junction transistors, P.I.R.E., décembre 1954, 42.

FERRIS (C. D.), A system for generating a stable d. c. baseline and suppressing noise when processing pulsed signals of physiological and other origin, Med. and Biol. Eng., 1966, 4.
GIORGIS (J.), Some large signal properties of planar and planar epitaxial transistors, G.E. Application, Note 90.13 .

CARRÉ (R.), Communication privée, article à paraître. 